\title{
Filigrane
}

Écoutes psychothérapiques

\section{Un nouveau regard sur la pulsion, le trauma et la méthode analytique. Deuxième partie : une théorie de la méthode}

\section{Wilfrid Reid}

Volume 17, numéro 2, automne 2008

L’avenir du clinicien II

URI : https://id.erudit.org/iderudit/019420ar

DOI : https://doi.org/10.7202/019420ar

Aller au sommaire du numéro

Éditeur(s)

Revue Santé mentale au Québec

ISSN

1192-1412 (imprimé)

1911-4656 (numérique)

Découvrir la revue

Citer cet article

Reid, W. (2008). Un nouveau regard sur la pulsion, le trauma et la méthode analytique. Deuxième partie : une théorie de la méthode. Filigrane, 17(2),

70-98. https://doi.org/10.7202/019420ar
Résumé de l'article

Une première partie de ce texte a présenté une nouvelle théorie de la psyché. Cette nouvelle théorie, dans la mesure où elle remet au travail le processus mémoriel, nous introduit, au plan de la méthode analytique, au nouveau paradigme de la transitionnalité en complémentarité avec le paradigme plus classique de la remémoration. Chemin faisant, nous posons un nouveau regard sur le transfert et l'interprétation. 


\title{
Un nouveau regard sur la pulsion, le trauma et la méthode analytique. Deuxième partie: une théorie de la méthode
}

\author{
wilfrid reid
}

\section{Liminaire}

Le parcours clinique de Wilfrid Reid l'a mené à la conceptualisation d'une nouvelle théorie portant sur la réalité psychique et la méthode psychanalytique. Dans le numéro Printemps 2008, nous avons publié la première partie de son parcours clinique, soit un texte élaborant sa théorie de la réalité psychique. Nous présentons ici à nos lecteurs la suite de son parcours clinique sous la forme d'un texte portant sur sa théorie de la méthode psychanalytique.

Une première partie de ce texte a présenté une nouvelle théorie de la psyché. Cette nouvelle théorie, dans la mesure où elle remet au travail le processus mémoriel, nous introduit, au plan de la méthode analytique, au nouveau paradigme de la transitionnalité en complémentarité avec le paradigme plus classique de la remémoration. Chemin faisant, nous posons un nouveau regard sur le transfert et l'interprétation.

«Si nous admettons que le quantitatif seul donne à un événement son caractère traumatique.»

(S. Freud, 1939, 100)

«Le passé est une poussière d'événements que nous interprétons.»

(Amin Maalouf, 2007)

«Point n'est besoin d'espérer pour entreprendre ni de réussir pour persévérer.»

(Guillaume d'Orange) 
$\mathrm{D}$ ans la première partie de ce travail, nous soulignons la pertinence de ne pas dissocier la théorie de la réalité psychique et la théorie du travail analytique. Telle sera notre conception de la psyché, telle sera notre conception de la méthode. Le présent texte s'emploie ainsi à revisiter les grands paramètres de la méthode analytique à l'aune d'un nouveau regard porté sur le fonctionnement psychique. Ce nouveau regard met l'accent sur l'activité psychique elle-même, ses modalités d'organisation, plutôt que sur les contenus de cette activité psychique. Cette métapsychologie des espaces psychiques entrera en complémentarité avec une métapsychologie plus courante qui est une métapsychologie de l'objet.

\section{Une nouvelle épistémologie}

Henri Atlan (1986) fait état d'une biologie nouvelle qui pose l'organisation de la matière comme l'objet véritable de cette discipline. Cette organisation de la matière vivante comporte nécessairement plusieurs niveaux d'intégration dans la mesure où un organisme vivant tel un humain se présente simultanément sous plusieurs aspects : il est à la fois un objet physique (atomique), biologique (macromoléculaire), cellulaire, physiologique, psychique, linguistique, social. La relation entre les divers aspects de cet objet devient alors une dimension fondamentale de la connaissance de cet objet.

Si chaque aspect de cet objet représente en lui-même un niveau d'intégration, la description d'un deuxième niveau d'intégration ne peut être traduite complètement dans le langage d'un premier niveau d'intégration. Par exemple, une meilleure connaissance de l'aspect physique concernant l'atome ne peut, d'aucune manière, invalider la pertinence du développement de nos connaissances quant à l'aspect biologique qui réfère au plan cellulaire. De fait, l'étude de la relation entre ces deux niveaux d'intégration demande la création d'un troisième niveau rendu nécessaire à l'intégration des deux premiers niveaux. Cette relation entre la physique et la biologie entraîne la création de la biologie moléculaire et il en est de même pour l'ensemble des niveaux d'intégration.

Une meilleure connaissance de l'aspect physiologique de l'humain n'invalide d'aucune façon la pertinence d'une meilleure connaissance du champ psychique grâce au développement de concepts d'ordre proprement psychologique... et ainsi de suite pour l'ensemble des niveaux d'intégration; chacun d'eux possède sa légitimité épistémologique propre. Une meilleure connaissance de cette réflexion épistémologique d'Henri Atlan nous préserverait sans doute d'un certain réductionnisme biologique de mauvais aloi qui malheureusement a cours trop souvent dans le milieu psychiatrique. Ne soyons pas dupes, nous prévient Henri Atlan $(1986,48)$ : «L'unité supposée de l'objet de connaissance ne signifie pas l'unité forcée de discours sur l'objet. »

On le voit: ce modèle épistémologique s'avère utile dans son champ d'origine en œuvrant à la création d'une nouvelle biologie. Nous proposons ici l'utilisation métaphorique de ce modèle en le déportant dans le champ de la psychanalyse: 
les diverses composantes de la psyché - système inconscient et système préconscient/conscient - représentent alors des niveaux d'intégration et la relation relativement harmonieuse ${ }^{1}$ entre ces deux niveaux demandera l'instauration d'un troisième niveau d'intégration, celui de la transitionnalité. Cette perspective épistémologique apparaît sous-jacente à une métapsychologie des espaces psychiques où l'organisation de la matière psychique devient l'objet véritable de la connaissance de cette matière en articulation certes avec une attention portée aux contenus véhiculés par cette matière psychique.

\section{La matière psychique}

Pour décrire l'organisation de la matière psychique, nous prendrons appui sur le postulat fondamental de la métapsychologie de Freud: la réalisation hallucinatoire du désir. Ce postulat stipule que dans l'organisation inconsciente, l'on ne peut distinguer «ce qui est vrai [ce qui relève de la réalité externe] et ce qui est une fiction investie d'affect [ce qui relève de la réalité interne]» (Freud, 1895, 191). Dans cette première organisation de la matière psychique, le rapport à la réalité externe est d'emblée problématisé dans la mesure où cette première perception de la réalité externe relève... de l'hallucinatoire.

Une intégration fonctionnelle de la réalité interne et de la réalité externe devient ainsi un enjeu fondamental du développement de la psyché. Notre théorie devra tenir compte d'une première structuration où l'hallucinatoire constitue le principe organisateur de la psyché; simultanément, cette théorie devra repérer les apports de la réalité externe susceptibles d'exercer une influence sur le destin de l'hallucinatoire. En un mot, nous aurons à concevoir un développement de la psyché qui tient en main les deux fils du structurel et du conjoncturel.

Du côté du structurel réside l'hallucinatoire, ce premier principe organisateur de la vie psychique; du côté du conjoncturel, nous retrouvons l'environnement et l'histoire de l'individu. Comment peut-on concevoir l'intégration du structurel et du conjoncturel? Dans un certain langage, la relation entre un premier niveau d'intégration d'ordre structurel et un deuxième niveau d'ordre conjoncturel demande la création d'un troisième niveau, celui de la «structure de la conjoncture». (Sahlins, cité par J. Press, 2007)

Qu'est-ce à dire ? Pour Winnicott, au point de départ, l'intrapsychique ne constitue pas par lui-même une entité fonctionnelle; celle-ci consiste dans l'ensemble individu/environnement. Cela signifie que dans la première organisation psychique inconsciente, il est une partie de cette première organisation qui, au plan métapsychologique, relève de la structure alors qu'au plan phénoménologique, pour l'observateur extérieur, elle relève de la conjoncture. Peut-on la désigner mieux que sous l'appellation de structure de la conjoncture? Le caractère paradoxal du concept nous situe au cœur de la pensée de Winnicott.

Structure de la conjoncture, disons-nous. Pour Winnicott, la réponse de l'objet constitue, en quelque sorte, le deuxième principe organisateur de la psyché. Au plan métapsychologique, il participe ainsi du structurel; au plan phénoméno- 
logique, il participe du conjoncturel; en effet, cette réponse de l'objet sera différente selon les aléas de la conjoncture qui préside à la rencontre du pôle individuel et du pôle environnement de cette première structuration qu'est l'ensemble individu/environnement. Au point de départ, les deux niveaux d'intégration que sont la réalité interne et la réalité externe sont confondues; selon les aléas de la structure de la conjoncture, sera ou non facilitée l'intégration de ces deux niveaux de par la création d'un troisième niveau d'intégration; la transitionnalité représente ce troisième niveau où nous sommes dans une séparation du dedans et du dehors et simultanément dans une réunion potentielle du dedans et du dehors. Pour Winnicott, l'espace potentiel permet la distinction sujet-objet; il réalise en même temps une réunion potentielle avec l'objet.

La constitution d'un psychisme individuel comme unité fonctionnelle implique ainsi une succession de logiques intégratives. Dans un premier temps, au sein du système inconscient, surgit une première logique intégrative psyché soma; elle concerne une première inscription psychique de la pulsion sous forme de motion pulsionnelle. Il s'agit d'un simple quantum d'investissement où la pulsion n'est pas encore liée aux mailles du réseau représentatif. Nous sommes en présence de «formes élémentaires du psychisme, entièrement traversées par des mobilisations dynamiques "motionnelles" liant le minimum de significations avec le maximum de mouvement, c'est-à-dire de tensions évocatrices d'une actualisation potentiellement agie»(Green, 2000a, 53). Cette appétence sauvage est d'autant plus terrifiante que le fait de la ressentir est en quelque sorte l'équivalent de l'agir; elle est inconsciemment associée à une perte de contrôle. Cette première inscription est l'œuvre de l'hallucinatoire pur, une modalité d'investissement qui accorde la primauté au quantitatif par rapport au qualitatif.

Dans un temps théorique postérieur, cette première organisation du système inconscient sous l'égide de l'hallucinatoire pur entrera en relation avec le deuxième organisateur du système inconscient, soit la réponse de l'objet. Lors d'une rencontre suffisamment bonne du pôle individu et du pôle environnement de l'ensemble individu/environnement, la réponse de l'objet, de l'ordre du plaisir éprouvé par cet objet, devra ne pas être trop éloignée de la projection du sujet, projection de son propre plaisir sur l'objet. Dans le système inconscient, l'hallucinatoire pur investira alors la représentation d'objet issue de l'expérience de satisfaction. Cette relation heureuse entre les deux principes organisateurs conuira à la création d'un troisième niveau d'intégration : la réalisation hallucinatoire du désir.

Nous sommes maintenant en présence d'une nouvelle logique intégrative où le système inconscient peut générer une inscription psychique de la pulsion qui lie celle-ci aux mailles du réseau représentatif. De par la réussite du paradoxe du trouvé/créé, l'expérience de l'omnipotence (Winnicott, 1971d, 129), en instaurant la réalisation hallucinatoire du désir, signe un premier arrimage de la réalité interne et de la réalité externe dans l'illusion de la création du monde par le sujet. Cette nouvelle organisation du système inconscient génère l'expérience de la satisfaction pulsionnelle sur le mode hallucinatoire. 
A contrario, lors d'une rencontre non suffisamment bonne psyché/environnement, la relation entre les deux principes organisateurs du système inconscient entraînera la création d'une nouvelle organisation de ce système sous la forme de la réalisation hallucinatoire du non-désir: l'impossibilité inconsciente de réaliser le désir devient la manière prépondérante de faire inconsciemment l'expérience du désir. De par la modalité hallucinatoire, cela prend la forme d'une constatation objective. La seule réalité extérieure que le sujet peut imaginer est celle qui fait obstacle à la réalisation de la satisfaction pulsionnelle. Tout autre aspect de la réalité extérieure fait l'objet d'une hallucination négative ${ }^{2}$. Au début de sa démarche thérapeutique, pour rendre compte de ses échecs dans sa vie amoureuse, la dame aux mille et une confidences — j'y reviendrai — me dira comment «les hommes sont incapables d'aimer». Seul le négatif est réel, nous dit Winnicott (1971a, 35).

De cette manière, nous pouvons décrire l'instauration progressive d'une succession d'espaces psychiques qui correspondent chacun à une logique intégrative : l'espace psychique de la motion pulsionnelle, puis l'espace psychique de la réalisation hallucinatoire du désir, ou encore son avatar négativant dans la réalisation hallucinatoire du non-désir. Ceci dit, si l'expérience de l'omnipotence, comme modalité organisationnelle du système inconscient, constitue un temps théorique important dans la construction d'un psychisme individuel, il n'en demeure pas moins que ce premier arrimage des réalités interne et externe prend forme dans le contexte psychique d'une indifférenciation de ces deux réalités.

Subséquemment, la réponse de l'objet, deuxième principe organisateur de la psyché, sera de nouveau mise à contribution en vue de la réussite du paradoxe du détruit/trouvé. Cette réussite est nécessaire pour articuler un premier niveau d'intégration, l'expérience de l'omnipotence où le lien avec la réalité externe relève du subjectif et un deuxième niveau d'intégration, l'épreuve de réalité où le lien avec la réalité externe relève de l'objectif. Dans le langage de Winnicott, c'est l'écart entre l'objet subjectif et l'objet objectif. Là encore, la relation heureuse entre ces deux niveaux d'intégration exige la création d'un troisième niveau; c'est l'accès à la transitionnalité. La différenciation de l'espace psychique du dedans et de l'espace psychique du dehors demande la création d'un troisième espace où la question n'est pas posée. S'agit-il du dedans ? S'agit-il du dehors?

Dans cette perspective, la transitionnalité devient une logique intégrative tierce qui permet d'intégrer les logiques intégratives propres des systèmes inconscient et préconscient/conscient. Le rapport entre ces deux systèmes peut dès lors prendre la forme d'une opposition/collaboration quand les processus tertiaires de liaison faciliteront les échanges entre les processus primaires et les processus secondaires. Advenant l'échec de la mise en place de la transitionnalité, le rapport entre les systèmes inconscient et préconscient/conscient ne peut opérer que sur le mode de l'opposition pure.

Notons-le au passage : la modalité du rapport entre les systèmes inconscient et préconscient/conscient est un facteur déterminant pour l'instauration d'un 
processus analytique; la nature de ce rapport importe davantage que le simple contenu du système préconscient, contenu auquel nous sommes souvent tentés de nous référer en clinique. Ainsi en est-il d'un patient qui, dans les premières rencontres, mettra de l'avant «le désir de se comprendre ». Nous sommes en présence d'un simple contenu préconscient/conscient qui, en lui-même, ne nous dit rien de la modalité du rapport entre les systèmes inconscient et préconscient/conscient. Si tant est que le processus analytique implique le passage de l'inconscient au préconscient/conscient, l'instauration de ce processus est d'abord redevable à la modalité initiale du rapport entre les deux systèmes.

Illustrons cliniquement ces propos. Une analysante débute le récit d'un rêve; elle décrit son engagement dans une scène érotique avec un cousin. Elle interrompt soudainement son récit; elle éprouve un malaise à le poursuivre. Cela n'est pas sans l'étonner car elle avait hâte de venir à la séance... précisément pour raconter son rêve. Avec la jonction du sexuel et du familial, le système inconscient, à l'évidence, met en scène un mouvement incestueux: le sexuel, ça se passe inconsciemment, en famille. En même temps, cette conflictualité inconsciente peut rencontrer une certaine traduction préconsciente/consciente dans une conflictualité interne entre le désir de raconter le rêve et le malaise associé au récit du rêve. Le préconscient/conscient peut indirectement, par certains dérivés, rendre compte de la conflictualité inconsciente en préservant le caractère interne de la conflictualité. L'étonnement de la patiente signe la présence d'une énigme située dans le monde intérieur, ce qui facilitera la mise en mouvement du processus élaboratif. Le rapport entre les systèmes inconscient et préconscient/conscient est un rapport d'opposition/collaboration.

Observons maintenant le traitement d'un contenu similaire dans une organisation psychique qui n'a pas accès à la logique intégrative de la transitionnalité. Ainsi en est-il de la dame au docteur. Nous la désignons ainsi car, un long moment dans la démarche thérapeutique, une question revient sans cesse en séance: «Qu'en pensez-vous docteur?» Au plan de l'organisation psychique, la patiente est confrontée à un vide affectif qui traduit une absence de représentation interne d'elle-même. Nous pouvons faire l'hypothèse d'une hallucination négative de son monde intérieur. Ce «sacrifice subjectal» (Green, 1990, 365) compromet toute perspective où elle serait en relation avec elle-même si ce n'est de manière vicariante à travers la relation avec autrui. Cette composante intrapsychique se manifeste au plan relationnel par l'incapacité d'être seule en présence de son thérapeute d'où la sollicitation constante de ce dernier.

La dame au docteur apporte un rêve où elle se marie avec son frère; dans le rêve, ce frère est devenu un cousin. Dans ses premières associations, du bout des lèvres, elle fait état de sa culpabilité : elle a entretenu une expérience incestueuse durant plusieurs années avec ce frère un peu plus âgé. La relation s'est poursuivie jusqu'à l'âge de 18 ans; longtemps, elle ne parvenait pas à dire non, assure-t-elle.

Au-delà de la culpabilité, retenons que, dans son discours, il est une seule association où elle s'exprime à la première personne. Elle dira: «Je ne veux pas que 
ce problème-là me suive toute ma vie». Nous pouvons ici observer que le seul contenu du système préconscient/conscient qui s'inscrit dans un mouvement d'appartenance psychique ne fait que prendre la simple contrepartie, l'exact contrepied du contenu du rêve: là où le rêve, avec le mariage, évoque symboliquement une union pour la vie, à l'état de veille, le contenu préconscient/conscient introduit une simple dénégation du contenu issu du système inconscient. Nous sommes dans un rapport de simple opposition entre l'inconscient et le préconscient/conscient: le préconscient ne fait qu'abolir le mouvement inconscient, il ne transpose d'aucune manière au plan conscient la conflictualité incestueuse inconsciente dans une conflictualité interne déguisée.

D'ailleurs, la dame au docteur présente son rêve sur un mode très dégagé affectivement. Nous tenterons d'attirer son attention sur le contraste entre sa position affective nocturne où elle marie son frère et sa position affective diurne où elle désire se dégager de cette relation incestueuse; le jour, il y a le non-vouloir et la nuit, un vouloir éternel. Toujours du bout des lèvres, comme une chose convenue, elle fait état d'un certain désir à l'endroit de son frère. Cette reconnaissance ne s'accompagne d'aucun malaise, le désir est d'ailleurs présenté sur un mode très secondarisé. Elle réfère maintenant à un certain savoir: on lui a rapporté que généralement, les personnes abusées s'attachent à leur abuseur. Nous observons ici un inconscient non pas refoulé, mais un inconscient clivé avec la mise en place d'une barrière bien étanche entre l'affectivité consciente et l'affectivité inconsciente: les éléments clivés ou dissociés font retour dans le sujet, à partir de l'extérieur, en l'occurrence à partir du discours d'autrui.

Dans cette modalité limite d'organisation psychique, le fonctionnement psychique en extériorité se manifeste également dans le système de causalité (Cahn, 2002, 63), l'absence d'une logique intégrative des systèmes inconscient et préconscient/conscient donne lieu à une causalité psychique externe. Toute mobilisation du sujet est vécue inconsciemment par le sujet comme prenant sa source hors de lui. En lieu et place de l'expérience affective déguisée du désir incestueux, la dame au docteur rend compte de la mobilisation inconsciente apparue dans le rêve en référant consciemment à ce qui se présente, à ses yeux, comme le horspsyché ; la poussée pulsionnelle abolie au-dedans revient du dehors.

Est-ce à dire qu'il est tout à fait innocent — étymologiquement, non nocere, non nuisible - d'être abusée par un frère aîné ? Sans doute, pas. En même temps, comment concevoir, au plus près des choses, les mécanismes psychiques qui, dans l'abus, pourront entraver le développement de la psyché ? Pour ce faire, il apparaît utile de tenir les deux fils du structurel et du conjoncturel. Certes l'abus du frère représente en lui-même un trauma relationnel. Par ailleurs, cet abus peut être considéré comme un symptôme illustrant le caractère dysfonctionnel plus large des relations interpersonnelles dans la famille; l'occultation familiale de l'abus apparaît comme un signe parmi d'autres à cet égard. Tel est, en quelque sorte, le trauma relationnel, soit la face diurne du trauma psychique. Ce trauma est de l'ordre du conjoncturel. 
Si nous articulons la part structurelle et la part conjoncturelle du trauma, le trauma relationnel devient l'avers dont le trauma structurel est l'envers. À ce titre, le trauma structurel est la face cachée du trauma relationnel. Qu'est-ce à dire ? Il est utile de rappeler ici le postulat fondamental de la métapsychologie de Winnicott: la réponse de l'objet participe de l'organisation première de la psyché. Au départ, il est toute une part du conjoncturel — la structure de la conjoncture qui est partie prenante de la première structuration de la psyché.

Ce conjoncturel, au plan phénoménologique, relève du structurel au plan métapsychologique ${ }^{3}$. La construction d'un psychisme individuel, c'est-à-dire le développement de la capacité de penser les pensées, exigera du sujet un travail psychique qui lui est propre, un travail qu'il est seul à pouvoir accomplir. Ce travail consiste dans l'effacement de cette part du conjoncturel qui, au plan métapsychologique, participe originellement du structurel. La réussite de ce travail du négatif est nécessaire pour que le sujet en vienne à penser sa souffrance psychique indépendamment du conjoncturel, indépendamment de l'environnement.

Ce travail d'effacement de la structure de la conjoncture est un travail qui a cours dans l'ensemble individu/environnement. Il devra être facilité par la conjoncture proprement dite: là se situe la fonction facilitatrice ou traumatique de l'environnement — c'est le trauma/environnement — selon qu'il s'inscrit ou non dans une rencontre suffisamment bonne psyché-environnement. L'environnement sera facilitateur dans la mesure où il favorisera la réussite de la double paradoxalité du trouvé/créé et du détruit/trouvé, partant l'instauration progressive de l'absence de l'objet, cette absence se situant à mi-chemin entre l'intrusion et la perte. Avec cette hallucination négative de l'objet, l'environnement, de par son effacement, prend place dans la psyché sous la forme de «l'illusion que l'objet n'est pas constitutif de l'appareil psychique »(Green, 1993, 381). Cette complémentarité du structurel et du conjoncturel autorise la mise en œuvre de la transitionnalité.

On le voit, la dame au docteur ne peut penser son expérience onirique et plus généralement sa souffrance psychique indépendamment de l'environnement, en l'occurrence indépendamment du comportement abusif de son frère. De fait, il est permis de s'interroger sur le rôle facilitateur de l'environnement primitif de la patiente en regard de son travail propre de négativation de l'hallucinatoire. On le sait, la réussite du paradoxe du détruit/trouvé demande que l'objet survive à la destruction de la part du sujet. En même temps, à toutes fins utiles, Winnicott désigne du terme de destruction toute activité du sujet qui sera accueillie comme pouvant détruire l'objet.

Une scène récente avec la mère ne peut que nous laisser songeur à cet égard; la dame au docteur rapporte une querelle survenue avec sa mère qui s'oppose farouchement à une nouvelle disposition du mobilier dans la chambre de la patiente. La mère aurait informé cette dernière que celle-ci aura toute latitude quant à l'aménagement de sa chambre... après la mort de sa mère. Nous pouvons certes nous demander dans quelle mesure l'environnement primitif a facilité la négativation de l'hallucinatoire alors que cette facilitation demande la possibilité de dire non à 
l'objet. À partir de cette scène récente, que peut-on imaginer de la possibilité pour l'enfant de dire non à maman?

Advenant l'échec de la négativation de l'hallucinatoire, l'appareil psychique ne peut intégrer la logique hallucinatoire du système inconscient et la logique nonhallucinatoire du système préconscient/conscient. La modalité hallucinatoire de l'investissement perdure dans la psyché lors de la mobilisation affective. Le système inconscient ne peut que transformer en son contraire la réalisation hallucinatoire du désir qui devient la réalisation hallucinatoire du non-désir. C'est l'abolition de toute poussée pulsionnelle qui serait issue du dedans; toute mobilisation pulsionnelle, pour le sujet, ne peut prendre sa source qu' au dehors.

Il est dorénavant une conflictualité inconsciente au sein même de l'organisation pulsionnelle entre la réalisation hallucinatoire du désir et la réalisation hallucinatoire du non-désir et cette dernière assume la primauté de l'organisation pulsionnelle. La dame au docteur semble, de la sorte, avoir aboli toute poussée pulsionnelle qui prendrait sa source au-dedans; si la patiente tente de se tourner vers elle, elle est d'emblée confrontée au vide intérieur, à la représentation de l'absence de représentation interne. Elle ne peut que se référer continuellement, systématiquement «au docteur».

Cette référence à une causalité externe représente une composante intrinsèque de son fonctionnement psychique; toute formation psychique, pensée, sentiment, comportement, ne peut être interprétée par elle que dans le cadre de cette causalité externe. Nous retrouverons ce système de causalité externe lors d'un retard inhabituel à une séance. La dame au docteur demeure avec sa mère. Pour justifier son retard, elle se met à vitupérer contre maman : celle-ci ne cesserait pas de lui confier des tâches qui apparemment se doivent d'être accomplies sans délai le matin même de la séance même si, la veille, la patiente se sera employée à la prévenir de n'en rien faire. Nous sommes bien en deçà de «l'illusion que l'objet n'est pas constitutif de l'appareil psychique» (Green, 1993, 381).

Soyons attentifs ici à la manière de penser l'obstacle à la rencontre avec le thérapeute. L'absence d'un appareil à penser les pensées signe une organisation psychique qui ne peut différencier la réalité interne et la réalité externe. Ce qui, au plan phénoménologique, fait partie de la réalité externe — la pensée de maman -, au plan métapsychologique fait toujours partie de la réalité interne: elle commande le comportement, elle n'est pas effacée à l'intérieur du sujet.

En même temps, ce surinvestissement de la frontière dedans-dehors a pour fonction d'occulter la conflictualité interne inconsciente; cette dernière s'avère inaccessible sous forme déguisée, dans le système préconscient/conscient. Peu auparavant, la dame au docteur a présenté un rêve où un homme s'emploie à lui «tirer des balles de fusil dans les cuisses et le bas-ventre». À l'évidence, la rencontre avec le thérapeute recèle un grand danger. Contrairement à ce que l'on observe chez la dame au récit malaisé du rêve, chez la dame au docteur, le conflit inconscient ne peut se traduire dans le préconscient par une angoisse qu'elle pourrait assumer personnellement; l'expérience émotionnelle d'un malaise dans la 
relation transférentielle est abolie; en lieu et place, nous avons un agir sous la forme d'un retard attribué à la mère.

\section{Les temporalités psychiques}

Si, au-delà des contenus psychiques, nous mettons l'accent sur l'articulation contenant-contenu, le développement de la psyché comporte l'instauration progressive d'une succession d'organisations de l'espace psychique de par l'actualisation d'une séquence de logiques intégratives. Cette métapsychologie des espaces psychiques est simultanément une métapsychologie des temporalités psychiques. De fait, les logiques intégratives qui président à la différenciation des espaces psychiques - espace psychique du dedans, espace psychique du dehors, espace transitionnel — sont également à l'œuvre dans la différenciation des diverses temporalités psychiques.

L'intégration réussie des temporalités psychiques est nécessaire pour l'accès à une prise de conscience qui possède véritablement une portée significative car «la prise de conscience n'est rien d'autre que la conscience du temps » (Green, 2000b, 175). Selon la formule consacrée, l'inconscient ignore le temps (Freud, 1915, 228). Certes l'inconscient se situe hors du temps linéaire. Il ignore la flèche du temps. Cependant «l'inconscient, ce sont les temps mêlés, ce n'est pas de l'intemporel» (Pontalis, 1986, 222).

La «conscience du temps» implique que le préconscient/conscient devienne porteur d'une autre temporalité que celle de l'inconscient en même temps qu'il intègre cette temporalité de l'inconscient. Le préconscient/conscient doit rendre opérationnelle une logique intégrative où les temps ne sont plus mêlés, où le passé précède le présent, qui précède lui-même le futur. Sur le modèle de l'espace transitionnel qui permet l'intégration des espaces du dedans et du dehors, l'articulation heureuse des temporalités propres aux systèmes inconscient et préconscient/ conscient demande la création d'un troisième niveau d'intégration où paradoxalement, les temps sont suffisamment mêlés pour assurer une actualisation affective vivante du passé et en même temps, les temps sont suffisamment différenciés pour assurer l'instauration de l'après-coup qui distingue le temps où ça se passe et le temps où ça se signifie. Ce temps de la transitionnalité permet l'expérience de la prise de conscience plutôt qu'une simple reviviscence hallucinatoire du passé.

\section{Le processus mémoriel}

Chemin faisant, cette évocation d'une temporalité transitionnelle, au-delà du contenu du souvenir, nous conduit à aborder le processus mémoriel lui-même. Le processus mémoriel intègre-t-il la logique temporelle des processus primaires où, dans la pensée magique, les rapports du passé sont vécus comme des rapports du présent (Freud, 1913, 100), et la logique temporelle des processus secondaires où les rapports du passé ne sont plus agissants dans le présent? Dans cette réflexion sur le processus mémoriel, il peut être utile de rappeler une distinction mise de l'avant par le philosophe Bergson cité par Paul Ricœur (2000). Bergson distingue 
une mémoire/habitude et une mémoire/souvenir: la mémoire/habitude concerne le processus mémoriel, sa modalité, alors que la mémoire/souvenir a trait au contenu psychique véhiculé par le processus mémoriel.

La compulsion de répétition serait-elle une modalité particulière du processus mémoriel? Dans le cadre de sa réflexion sur la réaction thérapeutique négative, Freud, en 1920, introduit la pulsion de mort et la compulsion de répétition. Dans ce contexte, il fera appel au modèle théorique de la névrose traumatique; dans le langage actuel, il s'agit du syndrome de stress post-traumatique. Dans la névrose traumatique, le rappel répétitif de l'événement traumatique constitue un trait marquant du tableau clinique. Cependant, le caractère fondamental de cette entité clinique a trait à la modalité mémorielle de ce rappel qui prend la forme d'une reviviscence hallucinatoire. La pensée portant sur le traumatisme est une pensée qui s'ignore comme pensée; celle-ci cesse d'être reconnue comme une pensée. Elle devient un phénomène sensoriel. Nous retrouvons dans le système préconscient/conscient un processus mémoriel qui est propre au système inconscient, soit l'hallucinatoire.

Prenant appui sur le modèle théorique de la névrose traumatique, nous pouvons considérer que le passé détermine dans l'actuel non seulement ce qui se dissimule derrière la chose présente, mais d'abord et avant tout, ce passé dissimule la modalité actuelle de regard sur la chose présente, y compris quand la chose présentée est le passé. En effet, c'est avec un regard qui informe/déforme le passé que je regarde le passé: "Le passé est une poussière d'événements que nous interprétons» (Maalouf, 2007). Une patiente dira avoir fait «une grande découverte». Elle a senti comment une très vive dramatisation est présente dans sa manière d'évoquer son passé. Elle dit se réjouir de cette découverte qui lui assure maintenant «un certain pouvoir» sur son passé. Il semble possible de prendre maintenant un peu de distance affective par rapport à une interprétation du passé dans un cadre de pensée magique où les rapports du passé sont vécus comme des rapports du présent. Nous observons un certain changement dans la modalité du processus mémoriel.

Au cours du développement psychique, l'intégration des temporalités inconsciente et préconsciente/consciente a pour effet de produire un processus mémoriel où, paradoxalement, les rapports du passé sont vécus comme des rapports du présent et, en même temps, ne sont pas vécus comme des rapports du présent. Cette temporalité propre à la transitionnalité exige la réussite du travail du négatif c'est-à-dire la négativation de l'hallucinatoire. Cette négativation s'avère nécessaire pour permettre la transformation d'un processus mémoriel de type hallucinatoire en un processus mémoriel de type transitionnel. À ce titre, la négativation de l'hallucinatoire devient un processus psychique déterminant pour la construction d'un psychisme individuel.

Dans cette ligne de pensée s'inscrit tout naturellement l'apport de C. Botella $(2005,22)$ présentant le concept de trauma au négatif ou trauma par défaut. Selon cet auteur, un phénomène psychique s'avère primordial pour un développement sain de la psyché. Dans le trauma par défaut, ce processus primordial n'a pas eu 
lieu. En l'occurrence, ici la préhistoire ne précède pas l'histoire, elle lui échappe. Dans ce trauma par défaut, c'est l'absence d'un processus psychique qui devient traumatique: selon nous, la négativation de l'hallucinatoire représente ce processus psychique primordial qui n'a pas eu lieu.

En ce qui concerne la mémoire/habitude, le processus mémoriel originel relève de la modalité hallucinatoire d'investissement, modalité propre au système inconscient; l'intégration des temporalités inconsciente et préconsciente/consciente exige la négativation de l'hallucinatoire. Cette négativation est nécessaire au cheminement d'une mémoire/habitude hallucinatoire à une mémoire/habitude transitionnelle, partant à l'instauration d'une temporalité transitionnelle. Cette dernière temporalité donne accès à l'expérience de l'après-coup qui est une condition sine qua non pour une prise de conscience mutative. En l'absence de l'expérience temporelle de l'après-coup, l'interprétation produira «un outsight» (Green, 2000b, 189), non pas un insight.

Dans l'après-coup, il est un écart entre «le temps où ça se passe et le temps où ça se signifie» (Green, 2000a, 45). Si cet écart est absent, toute évocation du passé s'inscrit nécessairement dans le cadre d'une pensée magique où les rapports du passé sont vécus comme des rapports au présent (Freud, 1913, 100). Cette manière d'évoquer le passé, loin de représenter une voie de dégagement, devient l'expression symptomatique d'un fonctionnement psychique traumatique dont le modèle théorique est la névrose traumatique. Ici, tout stimulus sensoriel apparenté de près ou de loin à l'événement ou l'environnement traumatique, conduit à une reviviscence hallucinatoire; le temps où «ça se passe» coïncide avec le temps où «ça se signifie». Dès lors, les faits et gestes des objets de l'environnement primitif exercent irrémédiablement une influence néfaste dans le présent comme dans le futur. «Les temps sont mêlés» (Pontalis, 1986, 222). Le passé est vécu comme imprimant une marque indélébile sur la psyché. Ici, tel chez la dame au docteur, le processus mémoriel conserve sa forme hallucinatoire originelle.

L'échec du travail du négatif fait en sorte qu'au plan d'une temporalité affective agissante, la totalité organisationnelle de la psyché - inconscient et préconscient/ conscient confondus - demeure régie par l'hallucinatoire. Avec la dérive négativiste du travail du négatif, l'hallucinatoire bascule simplement dans son contraire: la réalisation hallucinatoire du non-désir. C'est l'abolition de toute poussée pulsionnelle issue du dedans. Nous retrouvons le vide intérieur de la dame au docteur; ce vide intérieur, à ses yeux, prend valeur de réalité. La patiente ne peut que se référer au «docteur» pour toute pensée à propos d'elle-même ou encore se référer à autrui pour tout comportement qu'elle réprouve en elle. Ainsi à sa mère pour son retard en séance ou alors à son frère et aux hommes en général pour l'échec de sa vie amoureuse. Elle-même «n'existe pas» (Winnicott, 1970, 43).

Au passage, nous dégageons une nouvelle lecture de la pulsion de mort. La soidisant pulsion de mort se présente comme une modalité d'investissement qui prend la forme «d'une destructivité néantisante » (Green cité par Balier, 2005, 79). Cette destructivité ne comporte pas en elle-même une intentionnalité destructrice; elle 
constitue un avatar de l'hallucinatoire, ce premier organisateur de la psyché. Au sein même de l'organisation pulsionnelle, nous pouvons observer une conflictualité inconsciente entre la réalisation hallucinatoire du désir et la réalisation hallucinatoire du non-désir.

Une patiente fera écho à cette conflictualité inconsciente inhérente au mouvement pulsionnel. Elle rapportera avoir entrevu de manière très fugace, «comme dans un éclair», que «la vie peut être bonne». Pour elle, il s'agit d'une révélation: il serait possible que la vie soit «bonne», tout en comportant des épreuves, des contrariétés. Jusque là, la vie lui est toujours apparue comme essentiellement «pénible, souffrante»; et alors si l'on est très gentil, si l'on fait très bien les choses, on peut obtenir une petite récompense, de ci de là, un peu de répit. Voilà que la conflictualité inconsciente au sein de l'organisation de la vie pulsionnelle est remise au travail.

\section{L'oubli/souvenir}

Nous sommes ainsi amenés à distinguer deux modalités de souvenir, soit l'oubli/souvenir et le souvenir/trauma; dans le deuxième cas de figure, nous observons une zone traumatique de la mémoire. Une formulation de Claude Janin $(1990,973)$ est ici particulièrement heureuse :

«L'oubli et le souvenir sont les deux catégories de la mémoire; l'oubli est l'écran sur lequel se figure le souvenir, comme le souvenir est l'écran de l'oublié; l'un et l'autre vont ensemble indissociablement liés.»

Dans l'oubli/souvenir, le souvenir surgit sur un fond d'oubli; le souvenir devient le retour du refoulé. Le refoulement représente ici une composante dynamique dont le corollaire, au plan économique, quantitatif, s'avère la modalité transitionnelle d'investissement. Cette modalité d'investissement est elle-même la résultante d'un travail psychique sur le quantitatif qui facilitera l'entrée en scène du refoulement. Freud $(1939,100)$ nous dit comment «seul le quantitatif donne à un événement son caractère traumatique». Complétons la formulation: seul l'hallucinatoire, comme modalité d'investissement - un hallucinatoire non médiatisé par la transitionnalité - donne à une représentation quelle qu'elle soit son caractère traumatique dans la mesure où il $\mathrm{y}$ a alors entrave à l'intégration des processus primaires et secondaires. La capacité d'élaboration psychique est sérieusement compromise quand une représentation, au plan phénoménologique, devient une perception au plan métapsychologique. Dans cette éventualité, le clivage du moi (Freud) ou, dans le langage de Winnicott, la dissociation primaire devient le corollaire dynamique de la composante économique qu'est l'hallucinatoire non médiatisé.

Décrivons brièvement la présentation clinique du processus mémoriel selon qu'il prend une forme hallucinatoire ou transitionnelle. Dans le souvenir/trauma, 
nous repérons la présence de trois éléments. Un premier élément: de par l'actualisation de l'hallucinatoire, le caractère de vérité du souvenir, c'est-à-dire sa correspondance à la réalité externe, ne peut être mis entre parenthèse en vue d'élaborer sa résonance interne; le souvenir a d'ailleurs pour fonction d'occulter le dynamisme propre de la réalité interne. Le sujet se cramponne, en quelque sorte, à ce caractère de vérité, et ce d'autant plus facilement que très souvent, nous n'avons pas à douter de la réalité événementielle douloureuse qui est présentée : ainsi en est-il de la relation incestueuse de la dame au docteur.

Un deuxième élément: le contenu du souvenir réfère constamment à une situation très pénible, voire à une situation tragique. Enfin un troisième élément: dans le discours du patient, l'évocation du souvenir a inconsciemment pour fonction de mettre un frein au mouvement élaboratif. Ce souvenir, très souvent conscient, sera quasi systématiquement rappelé au moment où le patient est sur le point de prendre contact avec sa conflictualité interne inconsciente. Chez la dame au docteur, le rêve du mariage risque de faire surgir la conflictualité interne associée à l'investissement amoureux du frère. Le rappel du souvenir tombe dru ; il tue dans l'œuf toute amorce d'élaboration associative de l'investissement amoureux.

Dans l'oubli/souvenir, la présentation clinique est assez différente. La valeur de réalité du souvenir n'est pas primordiale; ce statut de vérité peut tout à fait bien être mis entre parenthèses. Un deuxième élément: il n'a pas nécessairement, tant s'en faut, un caractère pénible; le plus souvent, il a un caractère anodin; l'intérêt du souvenir est ailleurs. Enfin, un troisième élément: le souvenir s'inscrit volontiers dans un mouvement élaboratif. Il devient un simple maillon dans une chaîne associative qui se poursuit après son évocation. Loin de faire entrave à la reconnaissance de la conflictualité interne dans l'actuel, cet oubli/souvenir donnera parfois de la profondeur à une prise de conscience qui est en voie d'élaboration.

Il en est ainsi avec l'homme à la petite chatte blanche, évoqué dans la première partie de ce travail. Peu à peu, il semble amorcer une prise de contact avec une certaine destructivité néantisante comme modalité privilégiée d'investissement de sa poussée pulsionnelle. Auparavant, il était fort tenté, c'est même un euphémisme, de repérer comme relevant de la réalité extérieure tout obstacle à sa réalisation personnelle. Petit à petit, il devient davantage sensible à la présence en lui d'un mouvement massif de démission quand surgit un obstacle à sa réalisation pulsionnelle; son réflexe est d'emblée de baisser les bras. Dans l'élaboration de ce mouvement de démission, il rapporte un souvenir: il se rappelle une composition française rédigée à la fin du primaire où il décrit l'histoire d'un homme qui considère que sa vie est finie.

\section{Le modèle de la remémoration}

Le modèle de la remémoration représente le paradigme traditionnel de la méthode analytique. Dans ce modèle, la visée générale de la cure porte sur la levée 
de l'amnésie infantile. Il s'agit de tenter de dresser le tableau des années oubliées selon l'expression de Freud $(1914,107)$. Ce dernier, peut-on penser, est demeuré fidèle à ce modèle tout au long de son œuvre; il en viendra ultimement à élaborer le concept de construction qu'il avait déjà largement utilisé dans l'analyse de l'homme aux loups. La construction comme Freud l'entend à l'époque, cherche simplement à pallier les difficultés rencontrées dans la visée de remémoration, tout en demeurant inscrite dans cette visée.

L'expérience analytique ultérieure a montré que la remémoration en elle-même n'est pas suffisante pour induire un processus analytique. L'évolution postérieure de l'homme aux loups illustre cet énoncé clinique de manière pathétique. Nous sommes conduits à concevoir les rapports du présent et du passé tout autant, sinon davantage, en termes de contenants inconscients qu'en termes de contenus inconscients, fussent-ils refoulés. Il importe de tenir compte de la modalité d'activité psychique; pour ce faire, le travail devra s'attarder sur les aléas de l'intégration des divers espaces et diverses temporalités analytiques dans la mesure où la réussite ou l'échec de cette intégration n'est pas sans exercer une influence directe sur la nature du processus mémoriel qui s'actualisera en séance.

Selon la formule de Jacques Press $(2007,5)$ ici «le passé ne se dissimule pas derrière le présent, il donne son organisation au présent. » L'organisation, ce maître-mot quand nous portons notre attention sur la modalité d'activité psychique. Dans le fonctionnement psychique limite ou traumatique ${ }^{4}$, l'influence la plus délétère du passé se manifeste dans la modalité traumatique d'organisation actuelle de la conflictualité inconsciente. Une modalité dedans-dehors de la conflictualité inconsciente est mise de l'avant en lieu et place d'une conflictualité inconsciente intrapsychique; ce faisant, le conflit interne s'avère complètement occulté.

\section{Une mémoire fictionnelle}

L'expérience analytique nous conduit de cette manière à introduire un nouveau paradigme de la méthode analytique : le modèle de la transitionnalité. Ce modèle prend pour objet la modalité d'organisation actuelle de la conflictualité inconsciente; le thérapeute cherchera à mettre au travail cette «empreinte» du passé (Press, 2007, 5) sur l'activité psychique actuelle. La visée générale de la cure, en complémentarité avec la remémoration, porte sur l'instauration de la transitionnalité ; nous œuvrons au développement de la capacité de jouer (Winnicott, 1971c, 76). Ce faisant, nous favorisons le développement d'un processus souterrain de remise au travail du processus mémoriel. Processus souterrain, disonsnous, car il peut se dérouler totalement à l'insu du sujet; nous pouvons cependant en recueillir les fruits dans l'émergence progressive d'une nouvelle modalité d'organisation de l'activité psychique, qui fera une part plus grande à la reconnaissance du conflit interne. Cette remise au travail du processus mémoriel demande paradoxalement, de la part de l'analyste, une certaine prise de distance par rapport au modèle de la remémoration. Nous avons à nous dégager d'un certain 
historicisme de la méthode analytique, où l'écoute du thérapeute accorde une place démesurée à l'histoire du patient, au détriment de l'enjeu structurel.

Or, au plan de l'organisation psychique, cet enjeu est fondamental; il a trait à la grande difficulté du sujet à se penser lui-même, et partant, à penser sa souffrance psychique indépendamment du hors-psyché, que ce hors-psyché concerne le présent ou le passé. La reconnaissance de la conflictualité interne inconsciente est nécessaire pour l'établissement du caractère opérationnel du modèle de la remémoration. Il devient alors impératif que le thérapeute, dans un premier temps, puisse lui-même penser le patient et sa souffrance psychique dans une conflictualité inconsciente qui soit propre au patient, dès lors, indépendamment de tout hors-psyché présent ou passé.

Winnicott $(1971 \mathrm{~b}, 100)$ nous dit que «le théoricien doit tenir compte de l'environnement». Soulignons le fait que Winnicott nous parle du «théoricien» et non pas du thérapeute. On sait qu'il importe, en séance, d'oublier la théorie. Cet énoncé possède ici toute sa prégnance. Le théoricien, en effet, ne peut que reconnaître le rôle de l'environnement primitif dans la construction du psychisme individuel. En même temps, dans la mesure où ce rôle consiste fondamentalement à faciliter l'effacement de la participation de l'environnement dans le fonctionnement de la psyché, apparaitt l'exigence d'une docta ignorantia dans la manière, pour le thérapeute, de penser le patient.

Le thérapeute, à l'encontre du théoricien, se doit de développer une ignorance savante de la participation de l'environnement présent ou passé dans le fonctionnement psychique, afin de faciliter le travail du négatif, soit l'effacement de l'environnement. Il s'agit de favoriser le développement de «l'illusion que l'objet n'est pas constitutif de l'appareil psychique». L'objet transférentiel apporte ainsi une contribution, appelée à demeurer silencieuse, au travail psychique d'effacement de l'objet. La distinction sujet-objet et la capacité de penser les pensées seront tributaires de la réussite de ce travail psychique du patient, facilité par la contribution du thérapeute. Le paradigme de la transitionnalité prend le pas sur le paradigme de la remémoration quand nous sommes en présence d'un fonctionnement psychique qui illustre l'échec de l'intégration des diverses temporalités psychiques. Dès lors, «il est moins question de lever l'amnésie infantile que d'autoriser l'enfance à se constituer en mémoire fictionnelle», nous dira André Green (2000b, 220). Cette proposition théorique se traduira par un nouveau regard porté sur le rapport entre le présent et le passé. Selon le modèle de la remémoration, le thérapeute recherche en quoi le présent dissimule le passé. La direction de la cure va du présent au passé. Dans le modèle de la transitionnalité, l'empreinte du passé s'exprime d'abord et avant tout dans une modalité actuelle d'organisation psychique en extériorité. Le discours sur le passé gagne alors à être entendu comme une manière de dissimuler l'expérience transférentielle inconsciente actuelle.

La direction de la cure se voit inversée. Quand le patient fait état, par exemple, du rejet parental dans un contexte psychique d'évacuation du conflit interne, 
indirectement, il est en train d'aborder un rapport inconscient à son thérapeute. Dès lors, le ici et maintenant amène moins à s'interroger sur le là-bas et autrefois ; le là-bas et autrefois amène plutôt à s'interroger sur le ici et maintenant. Si la démarche analytique consiste essentiellement dans l'analyse du transfert, le modèle de la transitionnalité nous invite à revisiter les deux grands paramètres de la méthode analytique que sont le transfert et l'interprétation.

\section{Le transfert}

Dans le fonctionnement psychique en extériorité, de par l'évacuation du conflit interne, la voie classique de l'interprétation stricto senso du transfert, soit le lien avec les objets primaires, est sérieusement mise à mal car la simple remémoration des souvenirs oubliés ne peut, par elle-même, induire un processus analytique; elle ne peut favoriser la reconnaissance et l'élaboration du conflit interne. Ce phénomène est très apparent chez la dame au lapsus. Nous reviendrons sur ce lapsus qui permet de la désigner. Pour l'heure, signalons que la patiente consulte pour un état dépressif; les préoccupations suicidaires sont suffisamment intenses pour que, durant une certaine période, ses filles en viennent à communiquer toutes les deux heures avec elle. L'état dépressif est consécutif à une rupture avec le conjoint. Ce conjoint, fréquenté depuis plus de dix ans, est décrit comme un être dévalorisant et violent verbalement à son endroit. Plusieurs ruptures ont émaillé la relation. Cette fois, précise-t-elle, ses filles lui ont fait promettre qu'il n'y aurait pas de retour en arrière.

Lors du premier entretien, d'entrée de jeu, la patiente s'emploie spontanément à me présenter «les causes» de sa dépression. Celles-ci sont de trois ordres, assure-t-elle. D'abord une «cause immédiate »: il s'agit de l'attitude constamment dévalorisante du conjoint. Puis il est « une cause à moyen terme » : c'est l'existence d'une jumelle avec laquelle elle n'a plus aucun contact depuis une vingtaine d'années. Cette jumelle s'est toujours montrée très agressive, très dévalorisante à son endroit; la chose fut d'autant plus nocive, précise-t-elle, que sa famille a longtemps nié cette attitude agressive de la jumelle jusqu'à ce que la famille fasse elle-même les frais de cette agressivité. Enfin, il est «une cause à long terme»: il s'agit d'une relation qui fut toujours très difficile avec sa mère; cette relation fut ponctuée de diverses séparations très douloureuses.

En écoutant la patiente, je ne peux m'empêcher de songer au clinicien peu favorable à la théorie analytique qui entendrait ce discours. Il aurait probablement la tentation d'y voir une nouvelle preuve de la non-pertinence de l'approche analytique. Visiblement, ces «prises de conscience» en aucune façon ne protègent la patiente des effets délétères d'une séparation: elle ne peut s'empêcher d'être dépressive, voire même d'être taraudée par des pensées suicidaires. Bien sûr, le dynamicien quelque peu averti saura reconnaître la présence de «pseudo-insight» chez la dame au lapsus. Il s'agit d'un savoir non-savoir, un savoir dissocié de toute mobilisation affective. Avec un tel savoir, l'on peut tout à fait bien faire l'économie du non-savoir. Avec de tels amis, dit-on, il n'est pas nécessaire d'avoir des ennemis. 
Nous sommes ici en deçà de «la conscience du temps» en ce sens que nous pouvons postuler une non-intégration des diverses temporalités psychanalytiques. $\mathrm{Au}$ plan des contenus psychiques, le système préconscient/conscient présente des éléments qui, éventuellement, pourraient être porteurs de significations potentiellement mutatives. Éventuellement... si, dans l'intervalle, sa psyché s'est quelque peu engagée dans un travail d'intégration des diverses temporalités psychiques. Ce processus se manifesterait alors par une certaine reconnaissance, tout à fait inexistante pour l'heure, de la conflictualité interne. Dans l'intervalle, dans une partie dissociée du moi, au plan de l'articulation des temporalités, la totalité de la psyché — inconscient et préconscient/conscient confondus — relève d'une pensée magique où les rapports du passé sont vécus comme des rapports du présent (Freud, 1913, 100).

Le savoir de la dame au lapsus n'entrave d'aucune manière la compulsion de répétition; ceci n'implique pas que ce savoir soit dépourvu de tout intérêt pour le thérapeute, en particulier l'association entre la jumelle et l'ex-conjoint... à la condition d'inverser la flèche du temps où classiquement le présent dissimulerait le passé. Au plan de l'affectivité inconsciente, nous sommes en présence de «temps mêlés »: nous pourrons faire l'hypothèse qu'en évoquant le rapport à sa jumelle, inconsciemment, de manière indirecte, elle présente le rapport gémellaire à l'ex-conjoint. Nous postulerons l'existence d'une conflictualité inconsciente dans l'actuel, une conflictualité de l'ordre de l'indistinction sujet-objet, et ce, dans un contexte psychique où tout conflit interne est évacué.

À aucun moment, la dame au lapsus ne s'interrogera sur les motifs qui ont pu la conduire à poursuivre aussi longtemps une relation si peu satisfaisante. Si nous proposons ce questionnement à la patiente, sa réponse fuse: «Il y a là un mystère». Ceci, en soi, n'est pas vraiment étonnant: n'y a-t-il pas toujours une grande part de mystère dans notre attachement aux êtres et aux choses? Malheureusement, du même souffle, elle s'emploie vigoureusement à fermer la porte à toute exploration de ce mystère. Elle s'empressera d'ajouter: «ce mystère ne m'intéresse pas ».

Le discours, qu'il réfère au présent ou au passé, a pour fonction d'évacuer, dans le hors-psyché, toute conflictualité interne inconsciente dans l'actuel alors que, à l'évidence, à l'insu de la patiente, le maintien d'une relation tout à fait insatisfaisante avec un conjoint dévalorisant représente, pour elle, une nécessité vitale; ici la mort psychique et la mort physique semblent confondues. La rupture avec le conjoint ne peut que la plonger dans des préoccupations suicidaires incessantes. Lors du deuxième entretien, la patiente aura d'ailleurs un lapsus qui signera cette indistinction sujet-objet où la séparation est de l'ordre de l'impensable. Elle veut signifier qu'elle n'a pas l'intention de renouer avec l'ex-conjoint; elle dira: «Je ne veux pas retourner avec moi.» Nous touchons ici vraisemblablement à l'une des composantes de cette nécessité vitale d'une relation où autrui, en la disqualifiant, lui permet de se disqualifier elle-même, de disparaître tout en déposant dans le hors-psyché la source de ce mouvement de «destructivité néantisante». La 
rupture, portant atteinte à cette mesure défensive, perturbe profondément un certain équilibre émotionnel de la patiente, cet équilibre fut-il des plus malheureux. Une souffrance très bruyante fait parfois écran à une douleur psychique (Pontalis, 1976, 267). Avec la rupture, l'écran semble disparu.

\section{La polarité transfert contre-transfert}

L'objet transitionnel est « la première possession non-moi » (Winnicott, 1971a, 8). En deçà de la transitionnalité, nous demeurons dans l'indistinction sujet-objet: ainsi je fais partie de l'objet et l'objet fait partie de moi. Paraphrasant Winnicott, dans ce contexte psychique, nous dirons que le transfert n'existe pas : ce qui existe c'est l'ensemble transfert contre-transfert. Dans cette perspective, l'émergence d'une élaboration du conflit interne, processus qui prendrait le relais de l'évacuation de ce conflit, passera nécessairement par une élaboration préalable du contre-transfert. Le développement de la capacité symbolisante du transfert ne pourra que transiter par la modalité d'accueil de ce transfert de la part du thérapeute. Cette modalité d'accueil logera dans une position affective inconsciente du thérapeute qui n'est pas sans posséder un caractère paradoxal. En effet, le thérapeute s'autorisera à entrer dans une relation où l'on n'a cure de séparer le tien du mien. En même temps, dans un mouvement oscillatoire, il préservera suffisamment la distinction sujet-objet pour assurer la distance nécessaire à l'élaboration du contre-transfert.

Le contre-transfert devient alors l'enjeu fondamental de la cure. «Ils ne mourraient pas tous, mais tous étaient frappés », nous dit le fabuliste. Une analyse soutenue des écarts inévitables ${ }^{5}$ par rapport à cette position affective inconsciente paradoxale du thérapeute, permet de naviguer au mieux parmi les divers pièges contre-transférentiels qui jalonnent la démarche thérapeutique. Signalons schématiquement les pièges les plus courants. Un premier piège réfère à la réponse du thérapeute face au statut de la vérité qui se manifeste dans le transfert. Dans le transfert limite, devient vrai objectivement ce qui est vrai subjectivement: la vérité du dedans devient la vérité du dehors; si je me sens rejeté, je suis rejeté.

Une première tentation contre-transférentielle ira dans le sens de chercher plus ou moins subtilement à disqualifier ce statut de la vérité. Dans un premier cas de figure, le patient risque de vivre douloureusement le fait de ne pas se sentir « compris » au sens étymologique du terme où cum prehendere renvoie à être pris avec, être accueilli. Dans un autre cas de figure, peut-être plus délétère, le patient entrera inconsciemment en collusion avec cette disqualification de son statut de la vérité, s'engageant alors dans une démarche thérapeutique en faux-self. Nous sommes parfois amenés à rencontrer certains patients qui, après des années d'analyse, présentent toujours une affectivité primaire (Winnicott, 1945, 33), une affectivité toujours demeurée sous l'égide de l'hallucinatoire; dès lors, ressentir l'affect continue de représenter une expérience traumatique.

En regard du statut de la vérité propre au transfert limite, il est un autre piège qui est, en quelque sorte, l'envers du premier. Cette fois, l'abolition du mouvement 
oscillatoire dans le contre-transfert nous conduit à demeurer inconsciemment enfermés dans le statut de la vérité du transfert qui confond le fait et le fantasme. Il en est ainsi, pour le patient, dans sa manière d'être affecté par ses affects; plus nous nous éloignons de l'angoisse signal d'alarme, pour nous approcher de l'angoisse automatique ou l'angoisse catastrophe, plus le patient éprouvera inconsciemment son angoisse selon une modalité où penser un danger et actualiser la réalisation d'un danger sont équivalents. Par exemple, pour le patient, le fantasme de ne pas pouvoir survivre à l'évocation des pensées suicidaires devient une réalité. À notre insu, ce statut de la vérité peut devenir très contagieux.

Il est un autre piège courant: celui de ne pas se tenir suffisamment à distance par rapport à un certain pessimisme thérapeutique. Le thérapeute aura nécessairement à composer avec ce pessimisme personnel qui fera écho au négativisme du patient. Dans la problématique limite, il est une conflictualité inconsciente au sein même de l'organisation de la vie pulsionnelle, entre la réalisation hallucinatoire du désir et la réalisation hallucinatoire du non-désir. Le primat de la réalisation hallucinatoire du non-désir prend cliniquement la forme d'une conviction inconsciente - à laquelle le préconscient/conscient mal différencié ne peut que s'associer où la satisfaction pulsionnelle s'avère impossible. Cette conviction inconsciente apparaît au sujet comme une constatation objective. Là encore, nous butons sur la très grande force du fantasme quand il prend les couleurs de la réalité. La patience devient ici un atout essentiel. Tel un viatique, il peut être utile, dans ce contexte, de garder en mémoire une formule attribuée à Guillaume D'Orange : «Point n'est besoin d'espérer pour entreprendre ni de réussir pour persévérer.»

\section{L'interprétation}

L'interprétation représente l'outil privilégié du modèle de la remémoration; l'accent est mis d'emblée sur la prise de conscience. Dans le transfert limite qui est un transfert non-symbolisé, pour le patient, souvent il n'y a pas vraiment de sens à rechercher du sens. Quel sens cela aurait-il de rechercher du sens dans un monde intérieur qui n'est pas différencié du monde extérieur, un monde où le conflit interne n'existe pas? Sans renoncer véritablement à l'interprétation, il peut être pertinent de relativiser son usage en l'inscrivant dans une triple polarité: les polarités silence/interprétation, élaboration/interprétation et interprétation/construction.

\section{La polarité silence-interprétation}

Le maniement du silence du thérapeute constitue, de fait, un enjeu déterminant pour faciliter l'accès au travail interprétatif. Ceci dit, le silence, cette règle d'or de la méthode analytique, ne doit pas être confondu avec le mutisme (Green, 1979, 321). Il s'agit de faire silence sur le contenu manifeste du discours. Parfois l'enjeu du silence du thérapeute est d'emblée à l'avant-scène comme chez la dame au docteur. Est-il possible alors d'intéresser plutôt le patient à la difficulté d'élaboration qui est sous-jacente à la question posée ? Dans le fonctionnement limite, le patient fera l'impasse sur l'établissement d'un rapport avec lui-même. Il tentera 
inconsciemment de prendre contact avec lui, de manière vicariante, par l'entremise du rapport avec autrui. Nous référons ici à une interpellation explicite du thérapeute; celle-ci n'est, en fait, que la pointe de l'iceberg. Très souvent, c'est tout le discours du patient qui implicitement interpelle très vivement le thérapeute.

En effet, au fonctionnement réflexif qui implique un rapport de soi avec soi, le patient substitue un fonctionnement réflexe où tout le langage verbal et non-verbal devient inconsciemment une action qui appelle une réaction du thérapeute. La mise à mal de ce rapport action-réaction représente un préalable au mouvement élaboratif. La règle d'abstinence prend ici toute sa prégnance. Il importe que le thérapeute ne présente pas la réaction attendue si nous voulons avoir accès éventuellement à l'angoisse occultée par l'agir ou l'action psychique.

Il importe de reconnaittre la très grande force de la sollicitation du patient en vue d'entraîner le thérapeute dans cette modalité relationnelle qui prend la forme d'une action-réaction; dans cette modalité relationnelle, il n'existe aucun temps de latence. Je pourrais citer, à ce sujet, une petite expérience personnelle dans le cadre de certaines activités de formation. Quand les participants à ces activités sont invités à visionner des enregistrements vidéo de premiers entretiens dans le cadre hospitalier, les premiers commentaires porteront souvent sur le silence du thérapeute. Certains, tout en reconnaissant le bien-fondé de ce silence et son utilité pour la suite des choses, feront état très volontiers du malaise qu'ils auraient ressenti à observer ce silence. D'autres participants s'interrogeront sur le caractère potentiellement violent de ce silence. L'un et l'autre commentaires sont pertinents. Au plan subjectif, le silence du thérapeute se présente comme un défi contretransférentiel important. Au plan objectif, nous devons reconnaître que nous faisons violence au fonctionnement psychique du patient; nous mettons à mal ce fonctionnement en rendant inopérante une défense privilégiée contre une angoisse qui s'apparente volontiers à une angoisse catastrophe. Nous devons reconnaître que nous cheminons sur un chemin de crête: comment peut-on œuvrer à ne pas occulter cette angoisse tout en veillant à ce qu'elle demeure tolérable en vue de faciliter son élaboration?

\section{La polarité élaboration-interprétation}

Le fonctionnement psychique limite comporte un processus mémoriel de type hallucinatoire; cette modalité d'investissement propre au système inconscient infiltre le système préconscient/conscient; l'affect devient alors un indice de réalité de sorte que ressentir un danger est l'équivalent de l'actualisation de ce danger dans la réalité. C'est la mise en abyme de l'affect quand il devient dangereux de ressentir un danger. Cette modalité de métabolisation de l'affect qui renvoie à la manière d'être affecté par les affects peut rendre compte de l'impératif qui s'impose à la psyché, celui d'occulter l'angoisse surgissant au centre de l'appareil psychique en déportant l'investissement vers la périphérie. Nous observons un surinvestissement des aléas des rapports dedans-dehors comme si l'ensemble de la vie psychique se réduisait aux heurts avec l'environnement (Pontalis, 1975, 45). 
Cette perturbation affective primaire représente un obstacle majeur au travail analytique; en même temps, elle désigne en creux la visée de ce travail. L'affect éprouvé comme indice de réalité constitue la caractéristique fondamentale du trauma structurel. Winnicott décrit un fonctionnement psychique qui comporte le gel d'une situation de carence de l'environnement. Sur ce modèle, nous pouvons concevoir que le fonctionnement psychique traumatique présente, en sous-jacence au gel de la situation de carence, un gel de la situation d'élaboration du trauma structurel. Le gel de la situation de carence y devient l'avers dont le revers est le gel d'un travail psychique sur la négativation de l'hallucinatoire. La remise en jeu des processus conduisant à la transitionnalité impliquera donc un passage obligé par le dégel du trauma structurel, partant une réactualisation du traumatique où l'affect devient un indice de réalité.

Nous dégagerons de cette manière un premier temps de la démarche thérapeutique où l'accent portera moins sur la destination du mouvement psychique inconscient c'est-à-dire la prise de conscience. Le thérapeute aura intérêt à porter son attention plutôt sur les aléas du mouvement lui-même, c'est-à-dire les difficultés propres à l'élaboration psychique. Le thérapeute aura d'abord pour visée une remise en marche du processus élaboratif et au premier chef, si possible, une élaboration de la manière d'être affecté par les affects. Pour ce faire, un long travail de liaison précédera, puis souvent accompagnera le travail d'interprétation.

«Là où réside un grand danger, là réside ce qui sauve » (Hölderlin, 1993, 162). La reviviscence hallucinatoire de l'angoisse, sur un mode plus ou moins accentué, devient un passage obligé du processus analytique. Cette reviviscence signera le dégel du traumatique issu du trauma structurel; en ce sens l'on peut dire que la relation analytique sera traumatique ou ne sera pas. L'existence de crises transférentielles devient une partie intégrante de la démarche thérapeutique quand celle-ci renoue véritablement avec la vie psychique dormante sous la destructivité néantisante. «Le grand péril» ne pourra que se manifester dans la relation transférentielle. Au gré des aléas de la représentance, tantôt ce «grand péril » sera contenu dans la relation, tantôt surgira un «grand péril » pour la relation. La crise transférentielle qui met en danger la relation représente fréquemment un des avatars de l'élaboration transférentielle. En cherchant plus ou moins consciemment à éviter à tout prix la crise transférentielle, nous risquons de compromettre l'instauration d'un processus analytique.

\section{La polarité interprétation/construction}

Freud (1937) introduira le concept de construction devant les difficultés rencontrées dans la levée de l'amnésie infantile. Devant l'échec de la remémoration, le thérapeute est invité à proposer une construction concernant ce qui a pu se passer durant les années oubliées. L'analyse de l'homme aux loups (Freud, 1918) est l'illustration paradigmatique de l'utilisation de la construction; l'échec de cette analyse mettra en lumière les limites de la construction telle qu'elle est entendue par Freud. 
Winnicott $(1960,237)$ questionne à sa manière le modèle de la remémoration et plus spécifiquement le concept de construction :

«Le psychanalyste n'est d'aucune aide s'il dit à sa malade: "Votre mère n'était pas assez bonne... votre père vous a séduite... votre tante vous a laissé tomber." En analyse, les modifications se produisent lorsque les facteurs traumatiques pénètrent dans le matériel psychanalytique selon les modalités propres au patient et dans le champ de sa toute-puissance (mots soulignés par W.R.). Les interprétations qui provoquent des altérations sont celles qui peuvent être données en termes de projection. »

Nous présentons cette longue citation car elle est potentiellement très riche au plan du renouvellement de la méthode analytique. Elle représente un point d'orgue dans la pensée de Winnicott. Est ici impliquée la nécessité d'un travail psychique sur l'hallucinatoire - le champ de la toute-puissance dans le langage de Winnicott - afin que la psyché puisse avoir accès à «l'illusion que l'objet n'est pas constitutif de la psyché». Dans ce contexte d'un travail sur le trauma structurel, le sujet pourra reprendre la métabolisation du trauma/environnement, voire du trauma/événement dans un nouveau contexte d'appropriation subjective de son histoire personnelle qui ne fait plus entrave à la reconnaissance de sa conflictualité interne inconsciente. Les péripéties de l'existence du sujet peuvent lui apparaître comme des projections. Le sujet pourra repérer une conflictualité inconsciente des objets primaires qui fera simplement écho à sa conflictualité interne propre; cette conflictualité interne des objets primaires cessera d'être utilisée pour occulter la conflictualité interne propre au sujet.

Il est d'ailleurs une question que l'on peut se poser à propos de la construction. Celle-ci est-elle en mesure de produire le processus analytique ou plutôt peut-elle apparaître comme le produit du processus analytique? Je serais tenté de mettre l'accent sur la deuxième hypothèse. Illustrons cliniquement ces propos. Retrouvons la dame aux mille et une confidences que nous avons rencontrée dans la première partie de ce travail. Rappelons que cette appellation réfère à un mode relationnel privilégié de la patiente au début de sa démarche thérapeutique: elle devient très volontiers la confidente des personnes malheureuses de son entourage alors qu'elle est d'une réserve extrême à propos d'elle-même. Au départ, elle présente un fonctionnement psychique en extériorité : dans une première tranche de sa psychothérapie, pour rendre compte de l'échec de sa vie amoureuse, elle fera longuement état de l'opposition de sa famille alors que l'homme dont elle était amoureuse était de confession religieuse différente. Plus tard, l'évacuation au dehors de la conflictualité interne prendra un autre visage, elle pourra se condenser dans un énoncé : «Les hommes sont incapables d'aimer.»

La dame aux mille et une confidences - doit-on se surprendre - éprouvera, au départ, de grandes difficultés à se confier à son thérapeute. Elle doit prendre 
soin d'elle de façon vicariante en prenant soin d'autrui. Cette modalité relationnelle défensive est heurtée de plein fouet dans la relation thérapeutique; celle-ci ne peut que mettre à nu les inhibitions sous-jacentes à ce mode relationnel. Elle est rapidement confrontée aux dangers considérables associés au fait de véritablement exprimer son être. Ces dangers sont d'abord présentés sur un mode projectif $^{6}$. Elle fera référence au discours maternel : la mère dit ne pas comprendre qu'elle puisse demeurer seule dans une pièce avec un homme qu'elle ne connaît pas. Ce sera là l'amorce d'une longue élaboration transférentielle dans le hic et nunc des angoisses associées à une véritable rencontre avec autrui, une rencontre où elle serait partie prenante, où elle serait concernée, non plus simple spectatrice.

Cette élaboration transférentielle aura cours dans le transfert proprement dit, dans la relation avec le thérapeute, également dans le transfert latéral, dans la relation actuelle avec sa famille et sa mère au premier chef. Le passage du fauteuil au divan marquera le cheminement du travail analytique: l'apprivoisement des angoisses transférentielles rendra moins prégnante la nécessité de tenir constamment à l'œil son thérapeute. Cette nécessité s'atténuera au fur et à mesure de la reconnaissance progressive d'une conflictualité interne inconsciente, une conflictualité logée au cœur de l'être (Winnicott, 1970, 43). Émergera graduellement une culpabilité primaire : elle est le mal, il lui est interdit d'avoir une existence propre. Nous sommes en présence d'une culpabilité de séparation (Modell, 1988, 65).

Les facteurs traumatiques de l'ordre du trauma/environnement sont d'emblée apparents dans l'histoire de la patiente. Elle est l'aînée de sa fratrie et à l'évidence, la mère éprouve de grandes difficultés émotionnelles; à titre d'exemple, durant l'enfance de la patiente, la mère se réfugie régulièrement dans sa chambre quand des visiteurs se présentent à la maison. De façon constante, la dame aux mille et une confidences est invitée à prendre le relais de sa mère dans de nombreux contextes relationnels. Dans la famille, tout semble graviter autour de la nonreconnaissance d'une certaine «folie» maternelle qui, à l'occasion, s'exprimera par des comportements explosifs. Cette «folie» maternelle fait l'objet d'une loi du silence qui pèse lourdement sur le climat familial. À l'évidence, présence d'un trauma relationnel... qu'il s'avère heuristique de considérer comme l'avers dont le revers est un trauma structurel compromettant l'instauration de la transitionnalité et partant, la reconnaissance de la conflictualité interne. L'élaboration psychique des angoisses transférentielles dans le hic et nunc apparaîtra comme la part visible d'un processus dont la face cachée a trait à un travail psychique portant sur une médiatisation progressive de l'hallucinatoire, avec une remise en jeu des mécanismes psychiques conduisant à la transitionnalité. Cette instauration de la transitionnalité se traduira cliniquement par une capacité nouvelle, celle de la reconnaissance de la conflictualité interne. Cette reconnaissance s'exprimera d'abord dans l'actuel: nous avons là une condition préalable au caractère opérationnel du modèle de la remémoration.

La dame aux mille et une confidences pourra maintenant «présenter les facteurs traumatiques en des termes qui permettent de les interpréter comme des 
projections » (Winnicott, 1960, 238). À cet égard, il est un mot de la patiente qui me semble tout à fait indicateur du travail psychique accompli: «Je m'en rends compte: j'ai davantage pris soin de ma mère que j'ai pris soin de ma vie». La patiente peut maintenant ressentir comme venant d'elle-même un phénomène que nous pouvons objectivement concevoir comme prenant sa source dans l'environnement. Cette construction heureuse illustre la capacité nouvelle de la patiente d'entrer en contact avec elle-même sans nécessairement passer par autrui.

Cette construction de la patiente met à jour le processus souterrain qui a accompagné silencieusement la longue élaboration psychique des angoisses transférentielles. Ce processus souterrain a trait à un effacement suffisant de l'environnement primitif - ce trauma par défaut de son histoire - de sorte que si «l'objet s'avère constitutif de l'appareil psychique», maintenant la patiente négative suffisamment cet environnement pour développer «l'illusion que l'objet n'est pas constitutif de l'appareil psychique», mais se présente plutôt comme étant posé devant lui. Nous sommes en présence d'une reconnaissance de l'existence d'une conflictualité interne inconsciente. La patiente pourra retirer les bénéfices de cette reconnaissance au plan de sa vie personnelle et professionnelle. L'instauration de la transitionnalité confère une portée significative à la remémoration. Michèle Bertrand $(2007,36)$ le dira à sa manière :

«La refiguration du passé n'appartient pas au passé. Elle peut donc être tenue pour un indice de la transformation psychique qui se poursuit chez l'analysant.»

La construction formulée par la patiente a-t-elle produit le processus analytique ? Il est plutôt permis de penser qu'elle est le produit du processus analytique.

\section{Le glissement du travail interprétatif}

En inscrivant l'interprétation dans une triple polarité, nous la situons en tension dialectique avec d'autres composantes de la méthode analytique. Ceci dit, dans l'abord du fonctionnement psychique limite, l'interprétation elle-même peut être mise au travail. À cet égard, Pontalis $(1974,53)$ soulignera la pertinence d'un certain glissement du travail interprétatif. Dans sa forme classique, l'interprétation tente de dégager le contenu latent qui se dissimule derrière le contenu manifeste. Une question se pose : «Qu'est-ce qui se cache derrière le contenu manifeste?»

Dans une nouvelle mouture du travail interprétatif, l'attention porte davantage sur l'activité psychique que sur le contenu de cette activité. Une nouvelle question se pose : «Comment ça fonctionne là-dedans ?» Dans cette interprétation/miroir, nous tentons de refléter au patient sa modalité d'activité psychique. Ainsi une recherche effrénée de «comprendre » peut avoir pour fonction d'occulter une angoisse associée au non-savoir. Il s'agit là d'une résistance majeure au processus analytique. Winnicott (1971c, 77) met bien en évidence dans le squiggle comment l'acceptation du non-savoir est une condition préalable à la découverte d'un savoir mutatif: 
«La personne que nous essayons d'aider a besoin d'une nouvelle expérience dans une situation particulière. L'expérience est celle d'un état qui ne se donne pas de but.»

Comprendre de façon utile demande d'accepter d'abord de ne pas comprendre.

\section{Conclusion provisoire}

La caractéristique propre d'une œuvre littéraire n'est pas l'exactitude informationnelle ou l'exactitude référentielle du texte, elle porte plutôt sur la manière d'organiser les éléments du texte. En bref, c'est la qualité de la composition, du style qui fera la grandeur de l'œuvre.

Mutatis mutandis, il en est de même du matériel analytique. L'accès au travail interprétatif relève de la qualité de la composition, de la modalité organisationnelle de l'activité psychique, davantage que des contenus de cette activité. Dans cette perspective, s'inscrit tout un courant de la pensée analytique qui, dans le fonctionnement psychique, met l'accent sur l'articulation contenant/contenu. La transitionnalité devient alors la pierre angulaire d'une métapsychologie des espaces psychiques. Chemin faisant, nous ne pouvons faire l'économie d'une réflexion renouvelée sur la question du trauma psychique, et ce, en renouant avec le postulat fondamental de la métapsychologie de Freud : la réalisation hallucinatoire du désir.

Dans son rapport de Lisbonne, Bernard Brusset (2006, 1220) soulève une question fort pertinente; il demande si nous devons vraiment concevoir l'intrapsychique comme étant uniquement de l'interpersonnel anachronique. Implicitement, il nous met en garde contre une certaine régression de la pensée analytique qui développerait une nouvelle forme de la neurotica. On le sait, cette théorie traumatique de la névrose des premiers temps de la pensée de Freud met l'accent sur les scènes réelles de séduction; elle situe dans la réalité extérieure le centre de gravité de la théorie analytique.

Seule la prise en compte simultanée du structurel et du conjoncturel dans la problématique du trauma psychique nous permet de faire toute sa place au rôle de l'environnement et en même temps de préserver le grand apport de Freud qui, avec la découverte de l'hallucinatoire, situe dorénavant le centre de gravité de la théorie analytique dans l'intrapsychique. De par l'existence en nous d'un territoire régi par l'hallucinatoire, nous sommes essentiellement des êtres truffés de contradictions.

Un rapport suffisamment bon avec l'environnement nous permet au mieux — et c'est une différence de taille - un aménagement plus harmonieux de ces contradictions en facilitant paradoxalement l'appropriation subjective de ces contradictions.

L'hallucinatoire et sa médiatisation grâce au travail du négatif se présentent comme les maîtres mots d'une nouvelle théorie de la psyché. Cette médiatisation de l'hallucinatoire donne accès à la créativité. Winnicott $(1971,1971$ e, 208) la définira comme «une certaine coloration de l'attitude face à la réalité ». Cela 
permet d'aborder la réalité avec «un regard toujours neuf » qui exprime inconsciemment le sentiment que «la vie vaut la peine d'être vécue» (Winnicott, 1971b, 91). La psyché peut alors faire place à un ailleurs, un au-delà de la perception. Dans cet écart conception-perception, Winnicott inscrit l'ouverture d'un espace pour un travail interprétatif. Edmond Rostand cité par Yvan Audouard (2001, 12) avait souligné : «Ce soleil sans qui les choses ne seraient que ce qu'elles sont». L'hallucinatoire et sa médiatisation par la transitionnalité ne représentent-ils pas le soleil de la psyché?

\author{
wilfrid reid \\ clinique bois-de-boulogne \\ 1575, boul. henri-bourassa ouest, bureau 505 \\ montréal (québec) h3m 3 a9
}

\title{
Notes
}

1. Pour la psychanalyse, la conflictualité interne inconsciente est inscrite au cœur de la condition humaine. Nous pouvons, au mieux, nous approprier subjectivement cette conflictualité et œuvrer, dès lors, à un aménagement plus satisfaisant.

2. L'hallucination (positive) consiste en une perception sans objet; l'hallucination négative a trait à la nonperception d'un objet. L'une et l'autre réfèrent à l'hallucinatoire où, sur le mode de la régression formelle dans le rêve, les images prennent valeur de réalité.

3. La description métapsychologique d'un phénomène psychique porte sur l'insertion de ce phénomène dans une théorie du fonctionnement psychique inconscient.

4. Dans le cadre des présentes hypothèses, les termes de fonctionnement psychique limite et fonctionnement psychique traumatique sont des synonymes. En deçà de l'instauration de la double limite, la psyché ne peut exercer sa fonction propre, i.e. la capacité de penser les pensées ou de les reconnaître comme pensées. Nous sommes en présence du traumatique, i.e. l'actualisation du trauma structurel.

5. La neutralité bienveillante devient le point de repère; l'élaboration du contre-transfert prend pour objet les écarts inévitables par rapport à cette neutralité bienveillante, et ce, au plan de l'affect et/ou de la représentation.

6. Pour repérer la projection, il est une question que l'on ne doit pas se poser: en regard du discours du patient, estce que cela est vrai, i.e. correspond à la réalité extérieure? L'existence de la chose dans la réalité externe n'empêche d'aucune façon la projection. Celle-ci n'a rien à voir avec la perception : elle réfère essentiellement à la mise au dehors de ce qui est au-dedans. La présence de la chose au dehors n'entrave d'aucune manière la mise au dehors de ce qui est au-dedans.

\section{Bibliographie}

Atlan, H., 1986, À tort et à raison, inter-critique de la science et du mythe, Paris, Éditions du Seuil.

Audouard, Y., 2001, La Provence de village en village, avec des aquarelles de Pierre Pellet, Rennes, Éditions Ouest, France.

Balier, C., 2005, La pensée psychanalytique confrontée à la violence dans la société contemporaine, in Autour de l'œuvre d'André Green, Enjeux pour une psychanalyse contemporaine, sous la direction de F. Richard et de F. Urribarri, Paris, Presses universitaires de France.

Bertrand, M., 2007, Construire un passé, inventer du possible ?, Rapport au $68^{\mathrm{e}}$ Congrès des psychanalystes de langue française, Genève, Mai 2008, document de travail pré-congrès. 
Botella, C., 2005. Enjeux pour une psychanalyse de demain, in Autour de l'œuvre d'André Green, Enjeux pour une psychanalyse contemporaine, sous la direction de F. Richard et F. Urribarri, Paris, Presses universitaires de France.

Brusset, B., 2006, Métapsychologie des liens et troisième topique. Rapport au 66e Congrès des psychanalystes de langue française, Lisbonne, Revue française de psychanalyse, 5, Tome LXX, Spécial congrès, Décembre 2006.

Cahn, R., 2002, La fin du divan?, Paris, Odile Jacob.

Freud, S., 1895, L'esquisse pour une psychologie scientifique, in La naissance de la psychanalyse, lettres à Wilhelm Fliess: notes et plans 1887-1902, Paris, Presses universitaires de France.

Freud, S., 1913, Totem et tabou, in Euvres compltes XI, 1911-1913, Paris, Presses universitaires de France, 1998.

Freud, S., 1914, Remémoration, répétition et élaboration, in La technique psychanalytique, traduit de l'allemand par Anne Berman, 4e édition, Paris, Presses universitaires de France, 1972.

Freud, S., 1915, L'inconscient, in Euvres complètes XIII, 1914-1915, Paris, Presses universitaires de France, 1988.

Freud, S., 1918, À partir de l'histoire d'une névrose infantile, in CEuvres complètes XIII, 1914-1915, Paris, Presses universitaires de France, 1988.

Freud, S., 1920, Au delà du principe de plaisir, in Euvres complètes XV, 1916-1920, Paris, Presses universitaires de France, 1996.

Freud, S., 1937, Construction dans l'analyse, in Résultats, idées, problèmes 2, Paris, Presses universitaires de France, 1985.

Freud, S., 1939, L’homme Moïse et la religion monothéiste, Paris, Gallimard, 1986.

Green, A., 1979, Le silence du psychanalyste, in La folie privée, psychanalyse des cas limites, Coll. Connaissance de l'Inconscient, Paris, Gallimard, 1990.

Green, A., 1990, La folie privée, psychanalyse des cas limites, Coll. Connaissance de l'Inconscient, Paris, Gallimard.

Green, A., 1993, Le travail du négatif, Paris, Les Éditions de Minuit.

Green, A., 2000a, Le temps éclaté, Paris, Les Éditions de Minuit.

Green, A, 2000b, La diachronie en psychanalyse, Paris, Les Éditions de Minuit.

Hölderlin, F., 1993, Odes, élégies, hymnes, poésie, Paris, Gallimard.

Janin, C., 1990, Les souvenirs appropriés, Revue française de psychanalyse, Tome LIV, 1990.

Maalouf, A., 2007, Émission Contacts, Radio-Québec, automne 2007.

Modell, A.-H., 1988, Psychoanalysis, in A New Context, Madison, Connecticut, International Universities Press, Inc.

Pontalis, J.-B., 1974, Le psychisme comme double métaphore du corps, in Entre le rêve et la douleur, Paris, Tel. Éditions Gallimard, 1977.

Pontalis, J.-B., 1975, Naissance et reconnaissance du soi, in Entre le rêve et la douleur, Paris, Tel. Éditions Gallimard, 1977.

Pontalis, J.-B., 1976, Sur la douleur (psychique), in Entre le rêve et la douleur, Paris, Tel. Éditions Gallimard, 1977.

Pontalis, J.-B., 1986, La jeune fille, in Perdre de vue. Connaissance de l'inconscient, Paris, Tel. Éditions Gallimard, 1988.

Press, J., 2007, Construction avec fin, construction sans fin, Rapport au $68^{\mathrm{e}}$ Congrès des psychanalystes de langue française, Genève, document de travail, Mai 2008.

Ricœur, P., 2000, La mémoire, l'histoire, l'oubli, L'ordre philosophique, Paris, Seuil.

Winnicott, D.-W., 1945, Le développement affectif primaire, in De la pédiatrie à la psychanalyse, Paris. Petite bibliothèque Payot, 1983.

Winnicott, D.-W., 1952, Psychose et soins maternels, in De la pédiatrie à la psychanalyse, Paris. Petite bibliothèque Payot, 1983. 
Filigrane, automne 2008

Winnicott, D.-W., 1960, La théorie de la relation parent-nourrisson, in De la pédiatrie à la psychanalyse, Paris. Petite bibliothèque Payot, 1983.

Winnicott, D.-W., 1970, Vivre créativement, in Conversations ordinaires, Coll. Connaissance de l'Inconscient, Paris, Gallimard, 1988.

Winnicott, D.-W., 1971a, Objets transitionnels et phénomènes transitionnels, in Jeu et réalité, Coll. Connaissance de l'Inconscient, Paris, Gallimard, 1975.

Winnicott, D.-W., 1971b, La créativité et ses origines, in Jeu et réalité, Coll. Connaissance de l'Inconscient, Paris, Gallimard, 1975.

Winnicott, D.-W., 1971c, Jouer, l'activité créative et la quête du soi, in Jeu et réalité, Coll. Connaissance de l'Inconscient, Paris, Gallimard, 1975.

Winnicott, D.-W., 1971d, L'utilisation de l'objet et le mode de relation à l'objet au travers des identifications, in Jeu et réalité, Coll. Connaissance de l'Inconscient, Paris, Gallimard, 1975.

Winnicott, D.-W., 1971e, Post-scriptum, in Jeu et réalité, Coll. Connaissance de l'Inconscient, Paris, Gallimard, 1975. 